\title{
Clinical validation of an algorithm for rapid and accurate automated segmentation of intracoronary optical coherence tomography images
}

\author{
Yiannis S. Chatzizisis a, ${ }^{*, 1}$, Vassilis G. Koutkias ${ }^{\mathrm{b}, 1}$, Konstantinos Toutouzas ${ }^{\mathrm{c}, 1}$, Andreas Giannopoulos ${ }^{\mathrm{a}}$, \\ Ioanna Chouvarda ${ }^{\mathrm{b}}$, Maria Riga ${ }^{\mathrm{c}}$, Antonios P. Antoniadis ${ }^{\mathrm{a}}$, Grigorios Cheimariotis ${ }^{\mathrm{b}}$, \\ Charalampos Doulaverakis ${ }^{\mathrm{d}}$, Ioannis Tsampoulatidis ${ }^{\mathrm{d}}$, Konstantina Bouki ${ }^{\mathrm{e}}$, Ioannis Kompatsiaris ${ }^{\mathrm{d}}$, \\ Christodoulos Stefanadis ${ }^{c}$, Nicos Maglaveras ${ }^{\text {b }}$, George D. Giannoglou ${ }^{\text {a }}$ \\ a Cardiovascular Engineering and Atherosclerosis Laboratory, First Department of Cardiology, AHEPA University Hospital, Aristotle University Medical School, Thessaloniki, Greece \\ ${ }^{\mathrm{b}}$ Laboratory of Medical Informatics, Aristotle University Medical School, Thessaloniki, Greece \\ c First Department of Cardiology, Hippokration Hospital, Athens University Medical School, Athens, Greece \\ d Information Technologies Institute, Centre for Research and Technology Hellas, Thessaloniki, Greece \\ e Second Department of Cardiology, General Hospital of Nikaia, Piraeus, Greece
}

\section{A R T I C L E I N F O}

\section{Article history:}

Received 18 December 2013

Accepted 18 January 2014

Available online 24 January 2014

\section{Keywords:}

Optical coherence tomography

Image processing

Image segmentation

Method comparison study

\begin{abstract}
A B S T R A C T
Objectives: The analysis of intracoronary optical coherence tomography (OCT) images is based on manual identification of the lumen contours and relevant structures. However, manual image segmentation is a cumbersome and time-consuming process, subject to significant intra- and inter-observer variability. This study aims to present and validate a fully-automated method for segmentation of intracoronary OCT images. Methods: We studied 20 coronary arteries (mean length $=39.7 \pm 10.0 \mathrm{~mm}$ ) from 20 patients who underwent a clinically-indicated cardiac catheterization. The OCT images $(\mathrm{n}=1812)$ were segmented manually, as well as with a fully-automated approach. A semi-automated variation of the fully-automated algorithm was also applied. Using certain lumen size and lumen shape characteristics, the fully- and semi-automated segmentation algorithms were validated over manual segmentation, which was considered as the gold standard.

Results: Linear regression and Bland-Altman analysis demonstrated that both the fully-automated and semiautomated segmentation had a very high agreement with the manual segmentation, with the semi-automated approach being slightly more accurate than the fully-automated method. The fully-automated and semiautomated OCT segmentation reduced the analysis time by more than $97 \%$ and $86 \%$, respectively, compared to manual segmentation.

Conclusions: In the current work we validated a fully-automated OCT segmentation algorithm, as well as a semiautomated variation of it in an extensive "real-life" dataset of OCT images. The study showed that our algorithm can perform rapid and reliable segmentation of OCT images.
\end{abstract}

(c) 2014 Elsevier Ireland Ltd. All rights reserved.

\section{Introduction}

Intracoronary optical coherence tomography (OCT) is an evolving imaging technique that allows visualization of the coronary arterial lumen in real-time with a precision superior to intravascular ultrasound (IVUS) [1,2]. Many studies have shown the utility of OCT in assessing plaque features, such as the fibrous cap thickness, lipid core size, even presence of macrophages $[1,3,4]$. OCT imaging of the coronary arteries can be applied for the identification of vulnerable plaque morphology,

\footnotetext{
* Corresponding author at: Cardiovascular Engineering and Atherosclerosis Laboratory First Department of Cardiology, AHEPA University Hospital, Aristotle University Medical School, 1 Stilponos Kyriakidi Street, 54636 Thessaloniki, Greece. Tel./fax: + 302310 994837.

E-mail address: joc@med.auth.gr (Y.S. Chatzizisis).

1 The first three authors contributed equally.
}

assessment of plaque progression, quantification of in-stent neointimal hyperplasia, longitudinal follow-up of stent endothelialization and investigation of stent strut malapposition [5-8]. Further, OCT images can be fused with coronary angiography for three-dimensional (3D) reconstruction of the coronary arteries, enabling the assessment of 3D arterial morphology and local hemodynamic microenvironment [9].

Typically, the analysis of OCT images is based on manual identification of the lumen borders. However, manual segmentation is a cumbersome and time-consuming process, subject to significant intra- and inter-observer variability. As a result, detailed OCT image analysis is currently impractical for routine clinical use and for analysis of large-scale data sets from clinical trials. Automated OCT segmentation could play a key role in accelerating and standardizing OCT image analysis for clinical and research purposes. In real-life settings, automated segmentation of intracoronary OCT images would be challenging, as the images have a wide variety of artifacts, e.g. cardiac motion artifacts, stent struts, side 
branches and presence of blood and thrombi in the lumen that need to be identified and appropriately handled.

The aim of the present study was to present and validate a fullyautomated method for segmentation of intracoronary OCT images. The proposed segmentation algorithms were based on the processing of discrete morphological features of the OCT images. The validation of our segmentation methodology was performed in a large dataset of "realworld" OCT images using manual segmentation as reference.

\section{Methods}

\subsection{Study population and OCT image acquisition}

We studied 20 coronary arteries [LAD (left anterior descending artery), $\mathrm{n}=14$, LCX (left circumflex artery), $\mathrm{n}=1, \mathrm{RCA}$ (right coronary artery), $\mathrm{n}=5$; mean length $=39.7$ $\pm 10.0 \mathrm{~mm}$ ] from 20 patients who underwent a clinically indicated cardiac catheterization. Six of those arteries had a stent. The characteristics of the study population are outlined in Table 1. The Institutional Ethics Committee approved the study. All study subjects provided written informed consent for their participation in the study.

The OCT acquisition was performed with a Frequency Domain OCT imaging system (FD-OCT C7-XRT OCT Intravascular Imaging System, Westford, MA, USA). The pullback speed was $20 \mathrm{~mm} / \mathrm{s}$, the axial resolution was $15 \mu \mathrm{m}$ and the frame rate was 100 frames/s. Temporary blood clearance was achieved with contrast injection. The analysis scheme of the OCT DICOM images is shown in Fig. 1. In total, 3966 OCT images were acquired. For the purposes of the current analysis we selected one every two images in 15 patients and one every three images in 5 patients to account for gating issues resulting in 1812 images. Of those, 130 (7\%) images were excluded. By consensus between two OCT experts those images were non-interpretable due to incomplete visualization of $>180^{\circ}$ of lumen circumference as a result of the presence of blood or thrombus. Representative examples of the excluded images are provided in Supplemental Fig. 1. The remaining 1682 OCT images (including 308 frames from stented segments) were segmented manually, as well as with a fully-automated approach. A semi-automated variation of the fully-automated algorithm was also applied. To further assess the performance of our algorithm in stented areas we compared the fully-automated segmentation and semi-automated segmentation of the frames $(n=308)$ derived from stented segments with the manual reference.

Table 1

Characteristics of the study population $(n=20)$.

\begin{tabular}{|c|c|}
\hline Men & $14(70 \%)$ \\
\hline Age, years & $63.3 \pm 8.6$ \\
\hline \multicolumn{2}{|l|}{ Risk factors } \\
\hline Smoking & $10(50 \%)$ \\
\hline Hypertension & $16(80 \%)$ \\
\hline Hyperlipidemia & $15(75 \%)$ \\
\hline Diabetes mellitus & $6(30 \%)$ \\
\hline Family history & $10(50 \%)$ \\
\hline \multicolumn{2}{|l|}{ History } \\
\hline Prior myocardial infarction & $3(15 \%)$ \\
\hline Prior stenting & $8(40 \%)$ \\
\hline Chronic kidney disease & $0(0 \%)$ \\
\hline Stroke & $0(0 \%)$ \\
\hline \multicolumn{2}{|l|}{ Indications for catheterization } \\
\hline Stable angina & $4(20 \%)$ \\
\hline Unstable angina & $8(40 \%)$ \\
\hline Non-STEMI & $3(15 \%)$ \\
\hline STEMI & $5(25 \%)$ \\
\hline \multicolumn{2}{|l|}{ Medications } \\
\hline Beta blockers & $12(60 \%)$ \\
\hline Statins & $13(65 \%)$ \\
\hline ACE inhibitors & $4(20 \%)$ \\
\hline Angiotensin receptor blockers & $12(60 \%)$ \\
\hline Aspirin & $15(75 \%)$ \\
\hline Calcium channel blockers & $4(20 \%)$ \\
\hline \multicolumn{2}{|l|}{ Lipids } \\
\hline Total cholesterol, mg/dl & $193 \pm 40$ \\
\hline LDL-C, mg/dl & $112 \pm 40$ \\
\hline HDL-C, mg/dl & $42 \pm 11$ \\
\hline Triglycerides, mg/dl & $144 \pm 54$ \\
\hline
\end{tabular}

Continuous variable are expressed as mean \pm SEM and categorical variables as absolute numbers (\%)

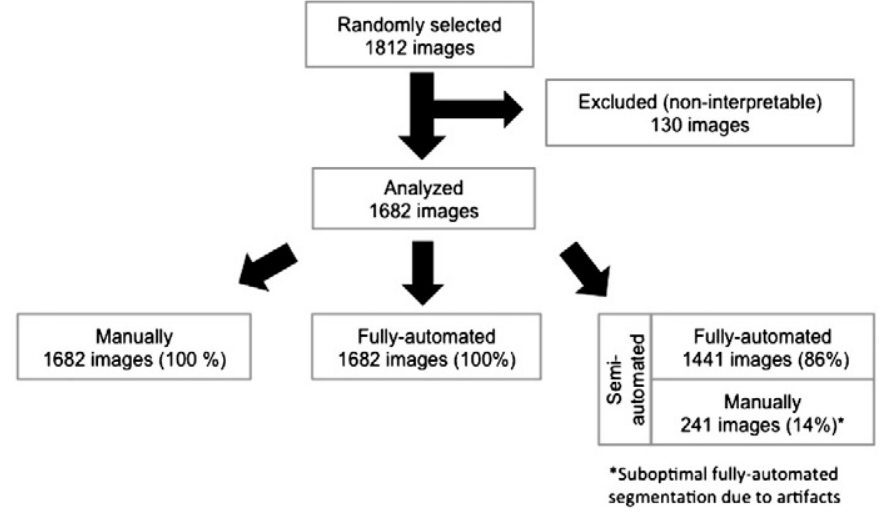

Fig. 1. Study design.

\subsection{Manual segmentation}

One independent OCT expert performed manual segmentation of the OCT images using an in-house application. The inter-observer agreement of manual segmentation was tested in a subset of 100 OCT images randomly selected from the entire dataset. These images were manually segmented by two independent OCT experts.

\subsection{Fully-automated segmentation}

The fully-automated OCT segmentation algorithm was developed in Matlab (The MathWorks, Natick, MA, USA) and is schematically presented in Fig. 2. By applying an iterative algorithm we used a threshold for transforming each image in binary form in order to distinguish the bright areas of interest. Morphological functions were applied to retain only those areas that were part of the wall. The lumen-wall contour in the radial scan was located at the point where the scan was identifying a bright shape (i.e. transition from black to white starting from the OCT catheter outwardly). In case of multiple transitions the correct transition corresponding to the actual lumen-wall border was determined with a heuristic approach. The steps utilized for the fully-automated segmentation of OCT images are presented below (Fig. 2):

i) Preprocessing: This involved several substeps applied to the OCT images, including gray-scale transformation, median filtering, smoothing, removal of calibration markers, transformation into polar coordinates, as well as removal of the OCT catheter.

ii) Main processing and extraction of the candidate contour points: Each OCT image was first transformed into a binary format by applying a local filtering scheme. Then, morphological filters were applied, such as an opening filter (erosion followed by dilation), which was used to eliminate all pixels in regions that were too small to contain the structuring element and a closing filter (dilation followed by erosion), which was used to fill-in holes and small gaps. These filters resulted in the extraction of the candidate contour points. Next, further heuristic rules and control mechanisms were introduced accounting for specific image characteristics that could affect the segmentation outcome.

iii) Segmentation of images with branches: Side branches, as well as other image artifacts were identified with statistical control of the contour gradient, taking into account the expected continuity of the contour. Outliers with respect to contour continuity were thus detected and appropriate corrections were done. Utilizing a heuristic method the lumen borders were found in images with large branches.

iv) Final outcome: Contour smoothing was performed in images in which the automated segmentation was suboptimal by applying a series of low-pass Haar filters [10].

\subsection{Semi-automated segmentation}

With the semi-automated approach all the images were initially segmented automatically with our algorithm as described above. After a review of the fully-automated segmentation by two independent OCT experts we performed manual adjustments in a subset of 241 images (14\%) due to suboptimal segmentation (Fig. 1). The major reason for suboptimal fully-automated segmentation was image artifacts, such as stent struts, presence of small amount of blood or thrombus in the lumen, and side-branches takeoff. Representative suboptimal segmentations are provided in Supplemental Fig. 2.

\subsection{Quantitative validation of fully-automated and semi-automated OCT segmentation}

The validation of the fully- and semi-automated segmentation algorithm was performed against manual segmentation, using certain vessel morphometric characteristics (Fig. 3):

i) Area $\left(\mathrm{mm}^{2}\right)$ of the identified lumen contour

ii) Minimum and maximum radius $(\mathrm{mm})$, representing the minimum and maximum length of the straight lines connecting the centroid of the detected contour with the lumen contour 


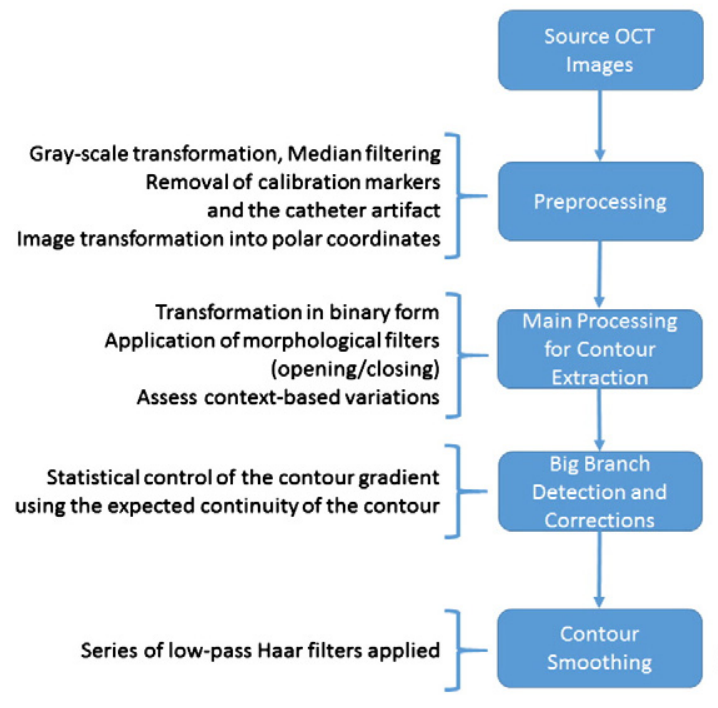

(A)

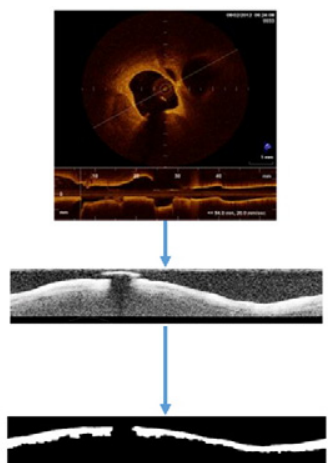

(B)

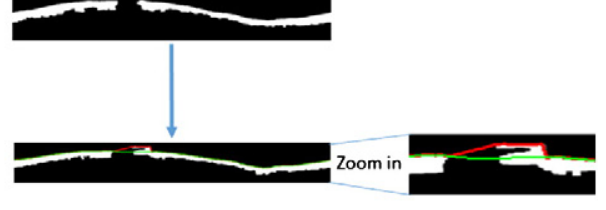

(C)

(D)

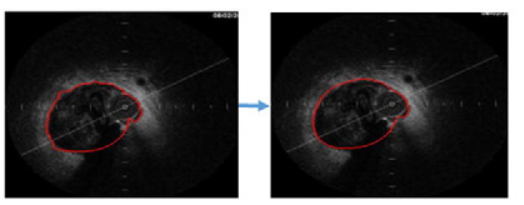

Fig. 2. Steps used in fully-automated segmentation of OCT images: A. Representation of polar coordinates of an OCT image, B. Image processing with application of morphological filtering and noise reduction, C. Candidate regions for contour correction; the corrections are depicted in green color, D. Final contour after Haar filtering.

iii) Minimum and maximum diameter ( $\mathrm{mm}$ ), representing the minimum and maximum length of the diameters that pass through the centroid of the detected contour

iv) Perimeter $(\mathrm{mm})$ of the lumen contour

v) Centroid $(\mathrm{mm})$ defined as the $\mathrm{x}$ - and $\mathrm{y}$-axis distance of the center of mass of the detected contour from the top left corner of the OCT image

The areas, radii and diameters were used as metrics of lumen size, whereas the perimeters and centroids were used as metrics of lumen shape.

\subsection{Statistical analyses}

Statistical analysis was performed with the statistical package GraphPad Prism 6.0 (GraphPad Inc., San Diego, CA, USA) and IBM SPSS Statistics 21.0 (IBM Corp., New York, NY, USA). All results were expressed as mean \pm standard error of mean. The quartiles of the variables were also calculated. The comparison of means among manual, automated and semi-automated segmentation was done with one-way ANOVA using Scheffe test to

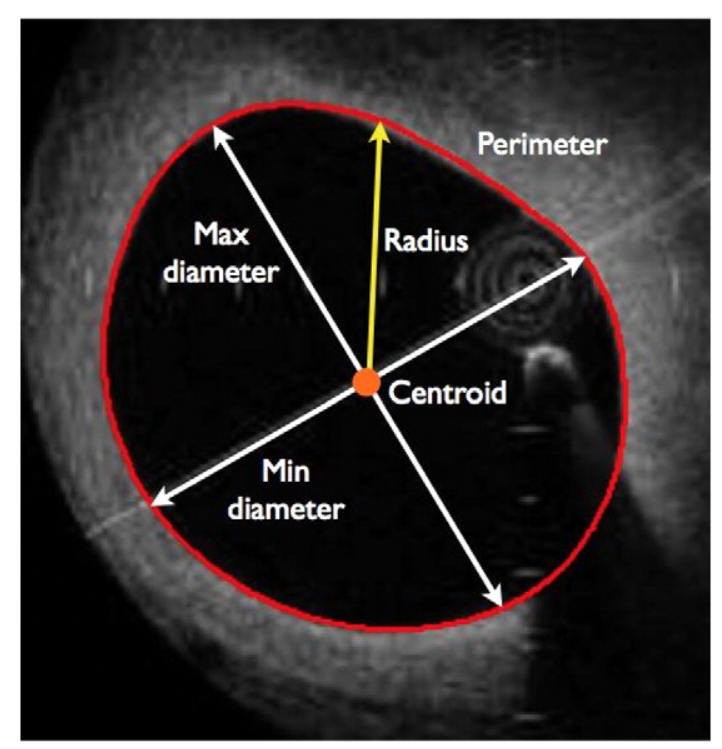

Fig. 3. Definition of the morphometric parameters utilized for the comparison of fullyautomated and semi-automated segmentation. correct for multiple comparisons. For the assessment of inter-observer agreement of manual segmentation linear regression analysis, Bland-Altman analysis and relative error calculation were performed. The relative error was calculated as the difference between two measurements over the reference measurement multiplied by $100 \%$. For the method comparison study between fully-automated or semi-automated vs. manual segmentation linear regression analysis and Bland-Altman analysis were performed [11]. In Bland-Altman plots the absolute differences between corresponding measurements ( $y$ axis) were plotted against the mean (x-axis). The mean differences (bias), as well as the limits of agreement (mean $\pm 1.96 \mathrm{SD}$ ), were calculated. The automated method was considered to agree with the manual measurements when the mean difference and the limits of agreement between the automated and manual analysis were comparable to the ones obtained from the inter-observer agreement of manual analyses. $\mathrm{P} \leq 0.05$ was considered as the level of significance.

\section{Results}

\subsection{Inter-observer agreement of manual segmentation}

The lumen area measurements of the two independent experts demonstrated a highly significant correlation ( $r>0.96$ for all the parameters) with high agreement (mean differences $0.0 \mathrm{~mm}$ or $\mathrm{mm}^{2}$ with narrow limits of agreement ranging from -0.9 to $+0.8 \mathrm{~mm}$ or $\mathrm{mm}^{2}$ for all the parameters, Table 2). The relative error for manual segmentations was low (limits from +0.1 to $+0.5 \%$ ). The increased interobserver agreement of manual segmentations justified the use of manual approach as gold standard for the validation of the automated algorithm.

\subsection{Validation of fully-automated OCT segmentation}

The fully-automated contour detection was validated versus manual segmentation in a large dataset of 1682 OCT images. Table 3 displays the quantitative data of the manual, fully-automated and semi-automated segmentation for all the morphometric variables. Fig. 4 shows the linear regression plots, the Bland-Altman analysis and the frame-by-frame analysis. The linear regression showed a significant association between manual and fully-automated segmentation, with slopes very close to 1.0 and intercepts close to 0.0 (Fig. 4A). The correlation coefficient was $>0.89$ for all the parameters with the exception of maximum radius 
Table 2

Inter-observer agreement of manual segmentation ( $\mathrm{n}=100$ images).

\begin{tabular}{|c|c|c|c|c|c|c|}
\hline & $\mathrm{r}$ & Linear regression equation & Bias & Limits of agreement & Relative error & $p$ \\
\hline Area $\left(\mathrm{mm}^{2}\right)$ & 0.99 & $\mathrm{y}=0.97 \times+0.15$ & 0.0 & $-0.9,+0.8$ & $0.2 \%$ & $<0.001$ \\
\hline Max radius $(\mathrm{mm})$ & 0.98 & $y=1.00 \times-0.01$ & 0.0 & $-0.2,+0.1$ & $0.5 \%$ & $<0.001$ \\
\hline Min radius (mm) & 0.96 & $\mathrm{y}=0.93 \times+0.07$ & 0.0 & $-0.2,+0.2$ & $0.3 \%$ & $<0.001$ \\
\hline Max diameter $(\mathrm{mm})$ & 0.99 & $y=0.98 \times+0.06$ & 0.0 & $-0.2,+0.2$ & $0.1 \%$ & $<0.001$ \\
\hline Min diameter (mm) & 0.98 & $\mathrm{y}=0.95 \times+0.11$ & 0.0 & $-0.3,+0.3$ & $0.0 \%$ & $<0.001$ \\
\hline Perimeter (mm) & 0.99 & $\mathrm{y}=0.98 \times+0.18$ & 0.0 & $-0.5,+0.6$ & $0.2 \%$ & $<0.001$ \\
\hline Centroid x (mm) & 0.99 & $\mathrm{y}=0.99 \times+0.04$ & 0.0 & $-0.1,+0.1$ & $0.2 \%$ & $<0.001$ \\
\hline Centroid y (mm) & 0.99 & $\mathrm{Y}=0.99 \times+0.02$ & 0.0 & $-0.1,+0.1$ & $0.2 \%$ & $<0.001$ \\
\hline
\end{tabular}

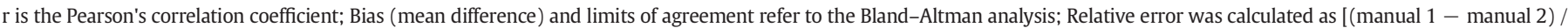
manual 1] $\times 100 \%$; $p$ refers to the Pearson's correlation coefficient and the linear regression analysis.

$(r=0.69)$ and maximum diameter $(r=0.86)$. In addition to linear regression, a high agreement between fully-automated and manual segmentation was found in the Bland-Altman plots showing mean differences close to zero (from -0.8 to $0.0 \mathrm{~mm}$ or $\mathrm{mm}^{2}$ for all parameters) and narrow limits of agreement (Fig. 4B). To further assess the agreement between fully-automated and manual segmentation, we plotted on the same plot each single OCT frame segmented with both methods (Fig. 4C). This head-to-head comparison showed a good agreement between the two methods. The relative error of fully-automated vs. manual segmentation for all the parameters ranged from -5.6 to $+0.2 \%$ and it was higher than the inter-observer relative error of manual segmentations. Regions where the fully-automated algorithm resulted in suboptimal segmentation mostly included side-branches take off and presence of stent struts (Supplemental Fig. 2), justifying the application of the semi-automated approach.
In stented segments Bland-Altman analysis showed that both fullyautomated and semi-automated segmentation had a high agreement with the manual approach (mean differences $<0.9 \mathrm{~mm}$ or $\mathrm{mm}^{2}$ with narrow limits of agreement, Table 4).

\subsection{Validation of semi-automated OCT segmentation}

Similarly to the fully-automated segmentation, the semi-automated method of contour detection was compared with manual segmentation. Linear regression analysis showed a significant association between the two methods that was stronger than that of the fully-automated vs. manual segmentation (Fig. 5A). The correlation coefficient was $>0.95$ for all the parameters. Similarly, Bland-Altman analysis revealed a higher degree of agreement between semi-automated and manual segmentation (Fig. 5B). Notably, the mean differences between these two methods

Table 3

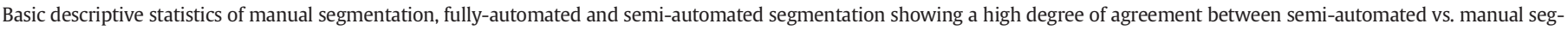

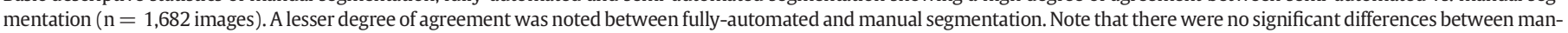
ual and semi-automated segmentation for all the parameters except for the perimeter.

\begin{tabular}{|c|c|c|c|c|c|c|c|c|c|c|c|c|c|}
\hline Parameter & & Min & $25 \%$ & Median & $75 \%$ & Max & Mean & SD & $\begin{array}{l}\text { Lower } 95 \% \text { CI } \\
\text { of mean }\end{array}$ & $\begin{array}{l}\text { Upper } 95 \% \mathrm{CI} \\
\text { of mean }\end{array}$ & $\begin{array}{l}\text { Manually vs. } \\
\text { fully-automated } p\end{array}$ & $\begin{array}{l}\text { Manually vs. } \\
\text { semi-automated } p\end{array}$ & $\begin{array}{l}\text { Fully-automated vs. } \\
\text { semi-automated } p\end{array}$ \\
\hline \multirow{3}{*}{$\begin{array}{l}\text { Area } \\
\qquad(\mathrm{mm})\end{array}$} & Manually & 0.5 & 3.9 & 5.3 & 7.8 & 14.8 & 5.9 & 3.0 & 5.8 & 6.0 & \multirow{3}{*}{0.327} & \multirow{3}{*}{0.884} & \multirow{3}{*}{0.607} \\
\hline & Fully-automated & 0.5 & 4.1 & 5.5 & 8.1 & 15.0 & 6.1 & 3.0 & 5.9 & 6.2 & & & \\
\hline & Semi-automated & 0.5 & 4.0 & 5.4 & 7.9 & 15.0 & 6.0 & 2.9 & 5.8 & 6.1 & & & \\
\hline \multirow{3}{*}{$\begin{array}{l}\text { Maximum radius } \\
\quad(\mathrm{mm})\end{array}$} & Manually & 0.5 & 1.3 & 1.5 & 1.8 & 2.7 & 1.5 & 0.4 & 1.5 & 1.6 & \multirow{3}{*}{$<0.001$} & \multirow{3}{*}{0.836} & \multirow{3}{*}{$<0.001$} \\
\hline & Fully-automated & 0.5 & 1.3 & 1.6 & 1.9 & 11.8 & 1.6 & 0.6 & 1.6 & 1.7 & & & \\
\hline & Semi-automated & 0.5 & 1.3 & 1.5 & 1.8 & 3.4 & 1.5 & 0.4 & 1.5 & 1.6 & & & \\
\hline \multirow{3}{*}{$\begin{array}{l}\text { Minimum radius } \\
\quad(\mathrm{mm})\end{array}$} & Manually & 0.3 & 0.9 & 1.1 & 1.4 & 2.0 & 1.1 & 0.3 & 1.1 & 1.1 & \multirow{3}{*}{0.957} & \multirow{3}{*}{0.137} & \multirow{3}{*}{0.072} \\
\hline & Fully-automated & 0.0 & 0.9 & 1.1 & 1.4 & 2.0 & 1.1 & 0.4 & 1.1 & 1.1 & & & \\
\hline & Semi-automated & 0.2 & 0.9 & 1.1 & 1.4 & 2.0 & 1.1 & 0.3 & 1.1 & 1.2 & & & \\
\hline \multirow{3}{*}{$\begin{array}{l}\text { Maximum } \\
\text { diameter (mm) }\end{array}$} & Manually & 1.0 & 2.4 & 2.8 & 3.4 & 5.0 & 2.9 & 0.8 & 2.8 & 2.9 & \multirow{3}{*}{$<0.001$} & \multirow{3}{*}{0.531} & \multirow{3}{*}{$<0.001$} \\
\hline & Fully-automated & 1.0 & 2.5 & 3.0 & 3.6 & 5.9 & 3.0 & 0.8 & 3.0 & 3.1 & & & \\
\hline & Semi-automated & 1.0 & 2.5 & 2.9 & 3.4 & 5.2 & 2.9 & 0.8 & 2.9 & 3.0 & & & \\
\hline \multirow{3}{*}{$\begin{array}{l}\text { Minimum } \\
\quad \text { diameter (mm) }\end{array}$} & Manually & 0.7 & 2.0 & 2.4 & 2.9 & 4.1 & 2.4 & 0.7 & 2.4 & 2.4 & \multirow{3}{*}{0.097} & \multirow{3}{*}{0.107} & \multirow{3}{*}{0.999} \\
\hline & Fully-automated & 0.6 & 2.0 & 2.4 & 2.9 & 4.2 & 2.4 & 0.7 & 2.4 & 2.5 & & & \\
\hline & Semi-automated & 0.6 & 2.0 & 2.4 & 2.9 & 4.2 & 2.4 & 0.7 & 2.4 & 2.5 & & & \\
\hline \multirow[t]{3}{*}{ Perimeter (mm) } & Manually & 2.8 & 7.2 & 8.3 & 10.0 & 13.7 & 8.5 & 2.2 & 8.4 & 8.6 & \multirow{3}{*}{$<0.001$} & \multirow{3}{*}{$<0.001$} & \multirow{3}{*}{$<0.001$} \\
\hline & Fully-automated & 3.0 & 7.7 & 9.1 & 11.1 & 19.9 & 9.3 & 2.6 & 9.2 & 9.4 & & & \\
\hline & Semi-automated & 3.0 & 7.6 & 8.8 & 10.5 & 14.8 & 8.9 & 2.3 & 8.8 & 9.0 & & & \\
\hline \multirow[t]{3}{*}{ Centroid x (mm) } & Manually & 2.7 & 4.2 & 4.6 & 4.9 & 6.1 & 4.5 & 0.6 & 4.5 & 4.5 & \multirow{3}{*}{0.009} & \multirow{3}{*}{0.044} & \multirow{3}{*}{0.853} \\
\hline & Fully-automated & 2.6 & 4.2 & 4.6 & 5.0 & 6.2 & 4.6 & 0.6 & 4.5 & 4.6 & & & \\
\hline & Semi-automated & 2.6 & 4.2 & 4.6 & 5.0 & 6.2 & 4.6 & 0.6 & 4.5 & 4.6 & & & \\
\hline \multirow[t]{3}{*}{ Centroid y (mm) } & Manually & 2.0 & 3.0 & 3.6 & 3.9 & 5.3 & 3.5 & 0.6 & 3.5 & 3.5 & \multirow{3}{*}{0.118} & & \\
\hline & Fully-automated & 2.0 & 3.0 & 3.6 & 4.0 & 5.3 & 3.5 & 0.6 & 3.5 & 3.6 & & 0.291 & 0.883 \\
\hline & Semi-automated & 2.0 & 3.0 & 3.6 & 4.0 & 5.3 & 3.5 & 0.6 & 3.5 & 3.6 & & & \\
\hline
\end{tabular}


A

Area

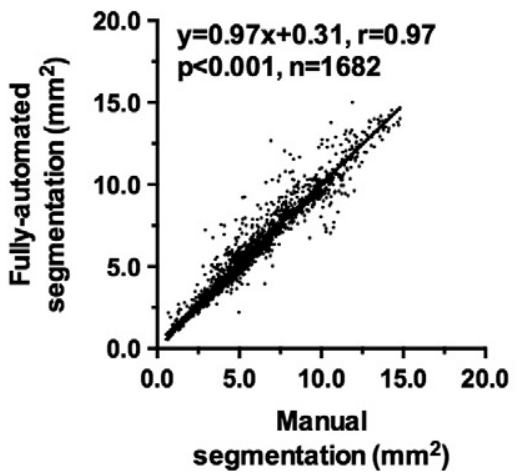

Max radius

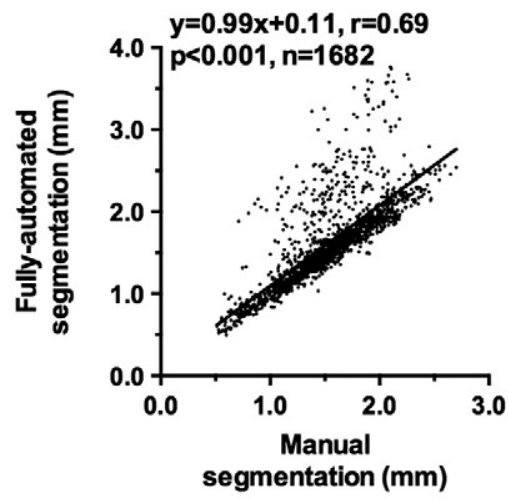

Max diameter

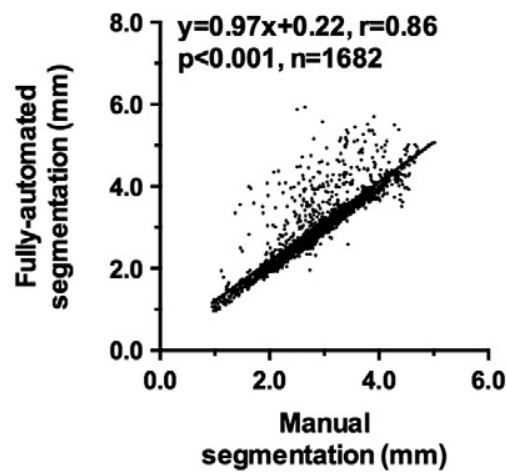

Centroid $\mathbf{x}$

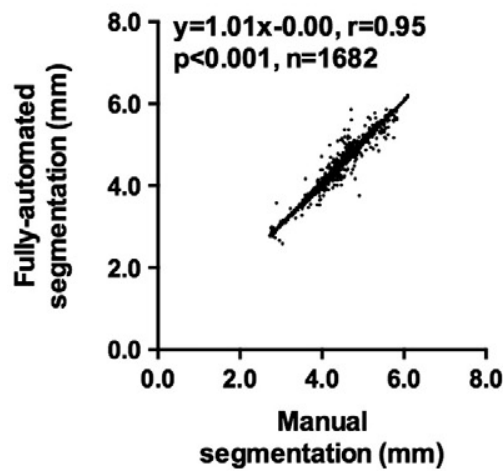

Perimeter

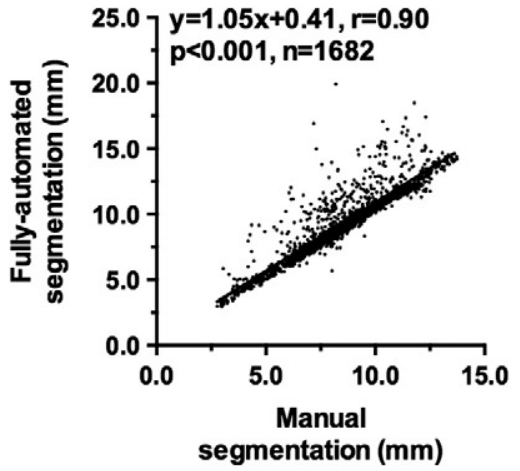

Min radius

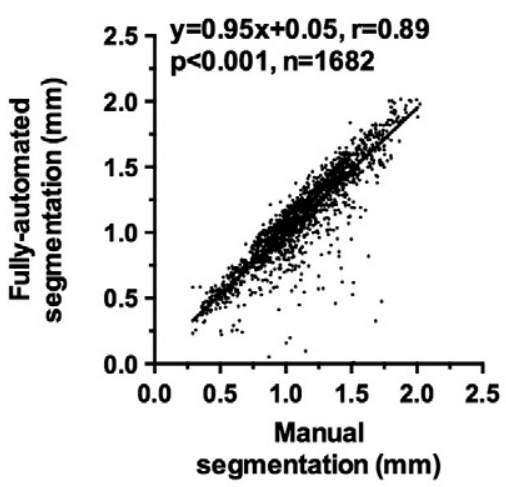

Min diameter

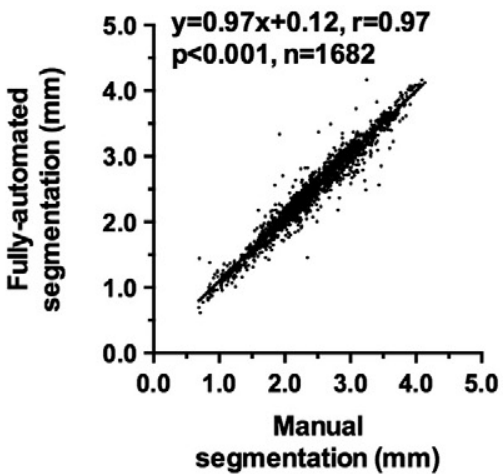

\section{Centroid y}

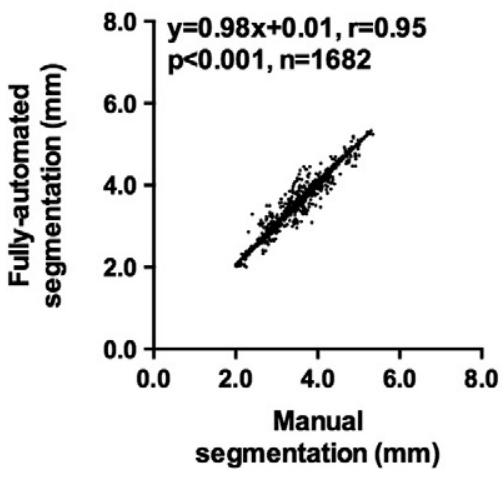

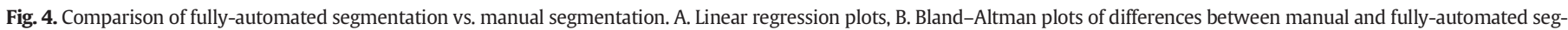

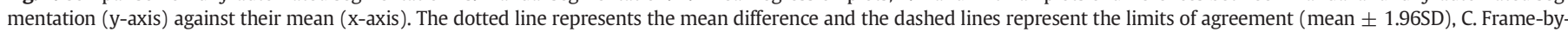

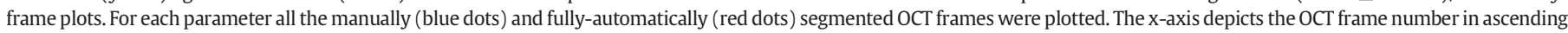
order, whereas the y-axis shows the corresponding morphometric variable. Note that the OCT images in x-axis are distributed independently of patient, type of artery and location. 
B Manually vs. Fully-automated: Area

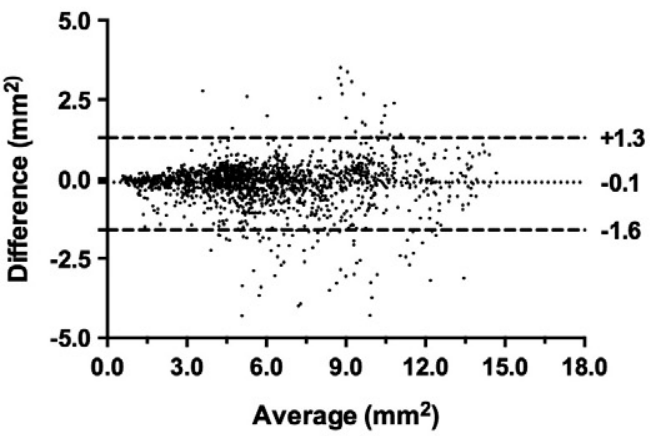

Manually vs. Fully-automated: Max radius

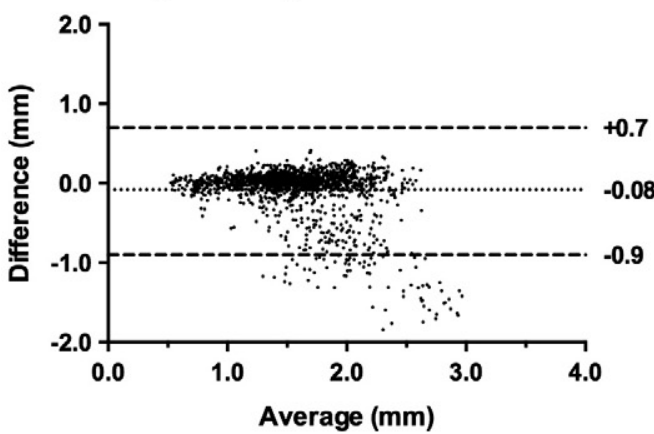

Manually vs. Fully-automated: Max diameter

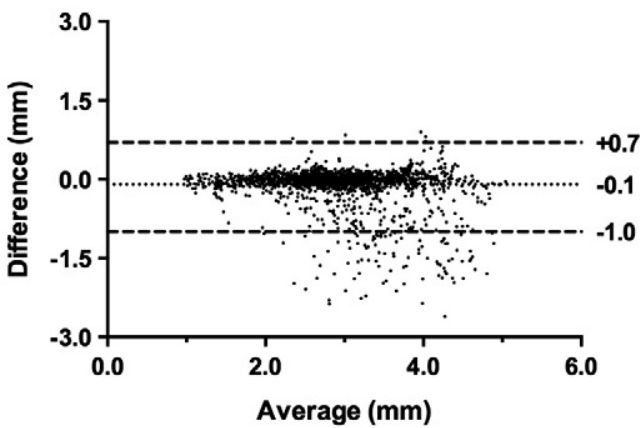

Manually vs. Fully-automated: Centroid $x$

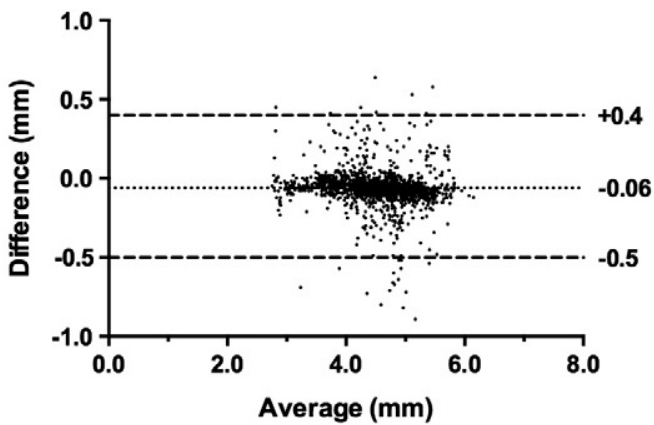

Manually vs. Fully-automated: Perimeter

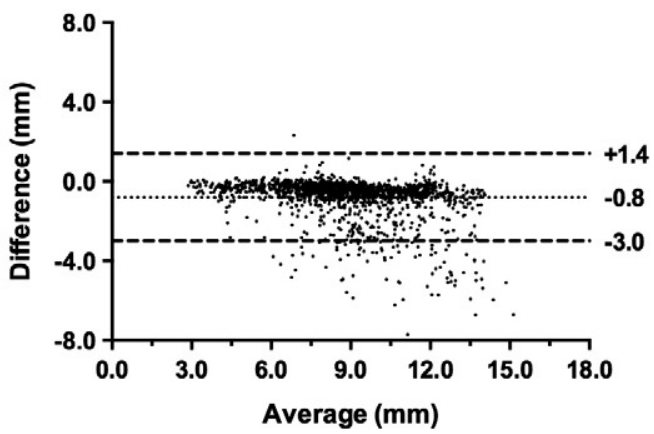

Manually vs. Fully-automated: Min radius

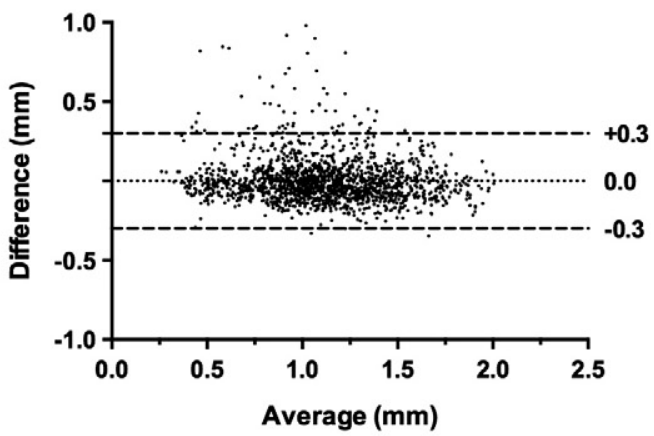

Manually vs. Fully-automated: Min diameter

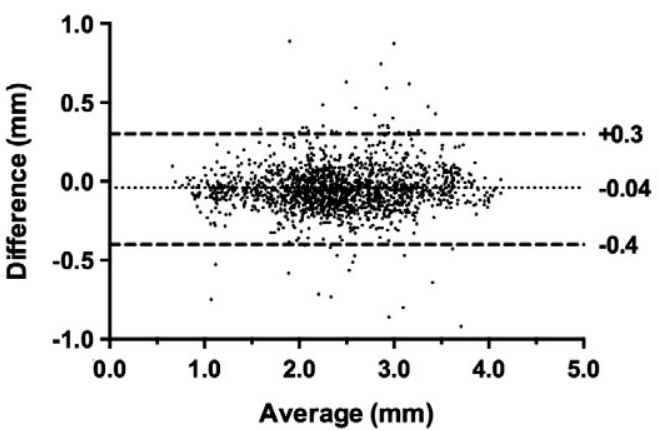

Manually vs. Fully-automated: Centroid y

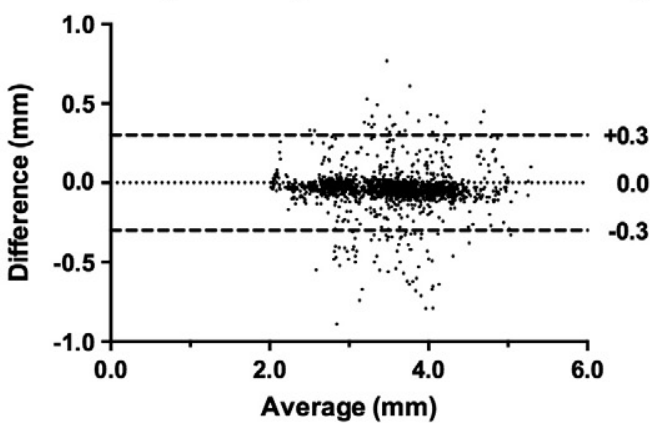

Fig. 4 (continued).

were even smaller (from -0.5 to $0.0 \mathrm{~mm}$ or $\mathrm{mm}^{2}$ for all variables) than with the fully-automated approach. This higher agreement was also demonstrated by the frame-by-frame plots (Fig. 5C), as well as by the relative error (limits for all parameters from -2.4 to $+0.3 \%$ ), which was comparable with the inter-observer relative error of manual segmentation.
Fig. 6A-C provides representative examples in which fullyautomated segmentation performed well, whereas Fig. 6D-F shows representative cases in which fully-automated lumen detection was suboptimal due to image artifacts. In these cases manual corrections had to be applied. 

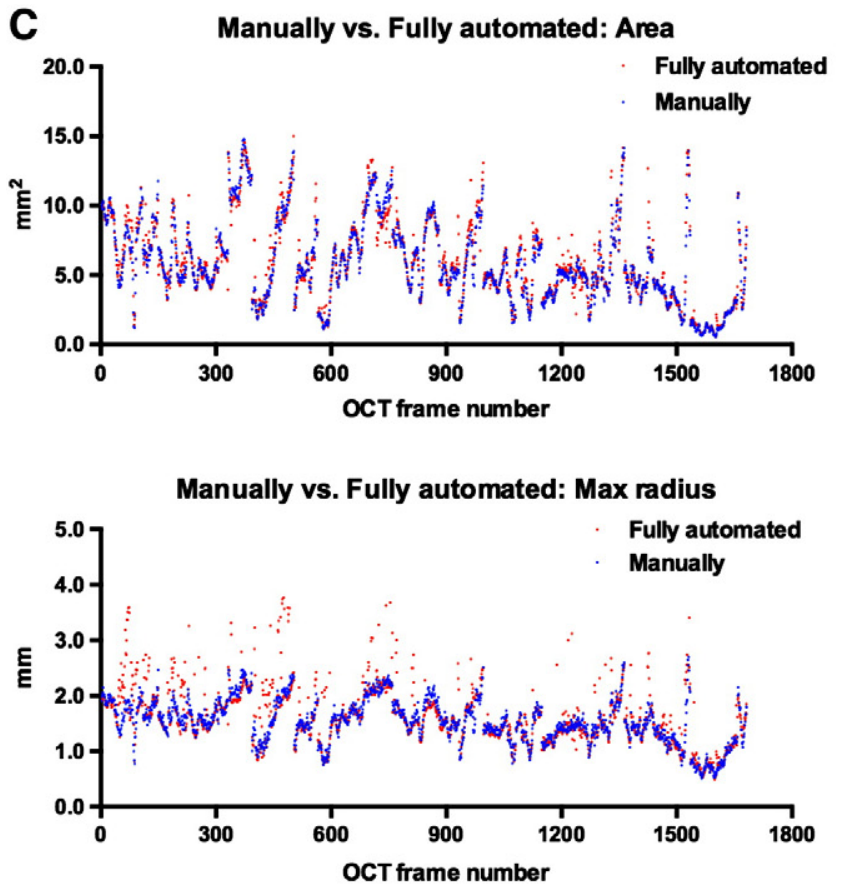

Manually vs. Fully-automated: Max diameter

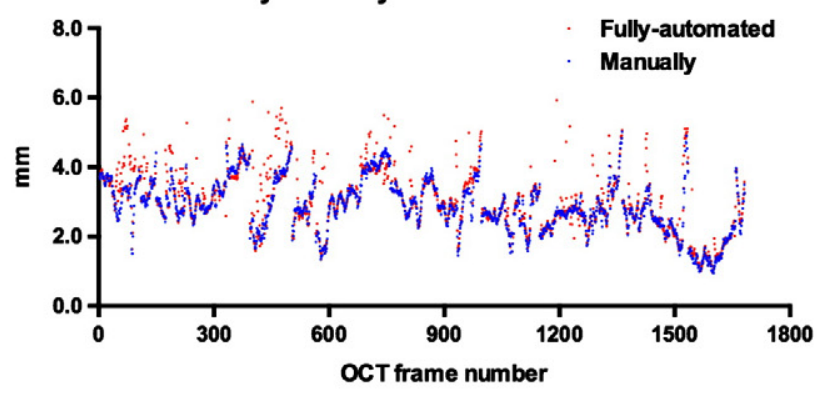

Manually vs. Fully-automated: Centroid x

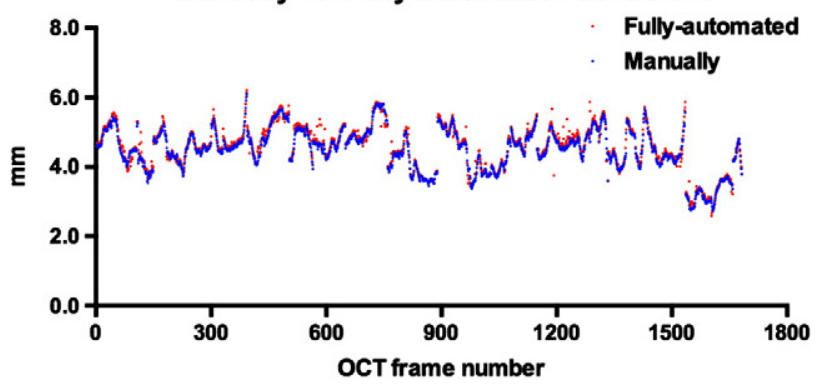

Manually vs. Fully-automated: Perimeter

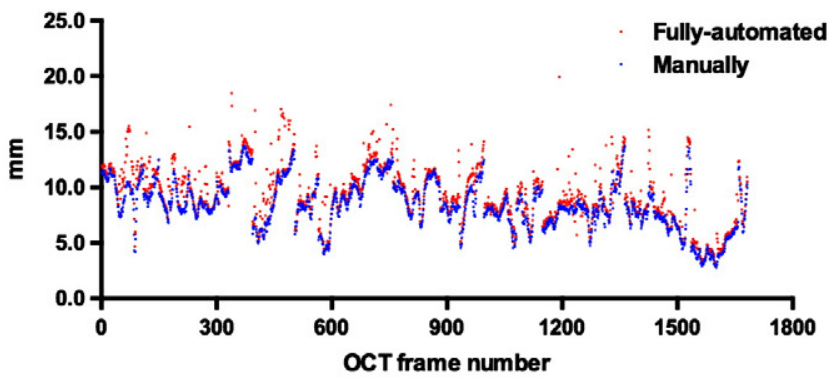

Manually vs. Fully-automated: Min radius

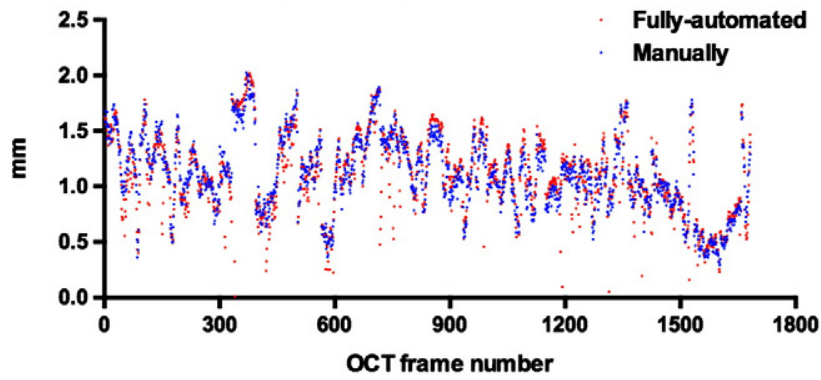

Manually vs. Fully-automated: Min diameter

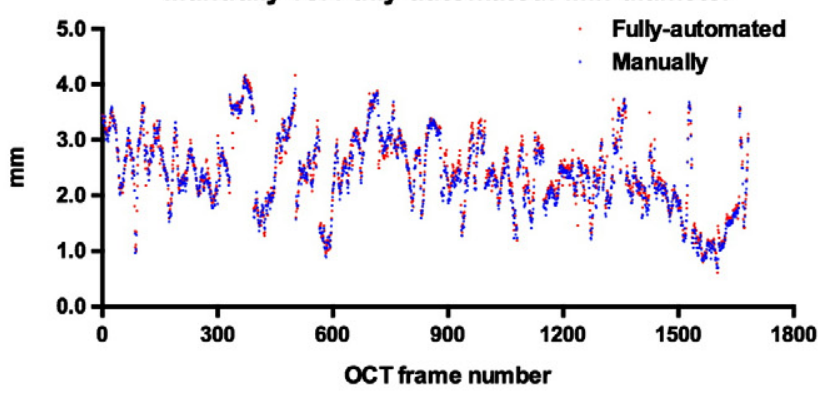

Manually vs. Fully-automated: Centroid y

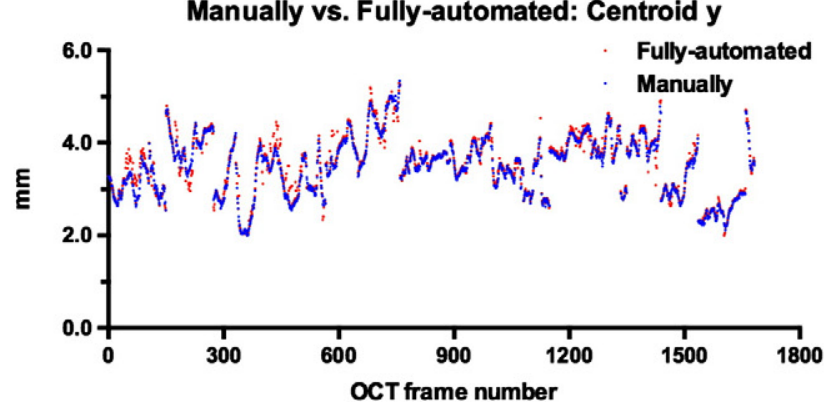

Fig. 4 (continued).

\subsection{Time gain of fully-automated and semi-automated OCT segmentation}

Table 5 displays the time gain of fully-automated and semiautomated OCT segmentation versus manual segmentation. Overall, manual segmentation required approximately $30 \mathrm{~s}$ of processing time per image, whereas fully-automated segmentation was done in less than $1 \mathrm{~s}$ per image and semi-automated segmentation in less than $5.1 \mathrm{~s}$ per image. As a result, the fully-automated OCT segmentation resulted in a time gain of more than $97 \%$. With the semi-automated segmentation there was more than $83 \%$ time gain compared with the manual approach. Of note, semi-automated segmentation improved the segmentation accuracy over the fully-automated method and at the same time it significantly decreased the analysis time over manual segmentation.

\section{Discussion}

In this work we presented a framework for automatically detecting the lumen contours in intracoronary OCT image sequences. We validated our algorithm in a large dataset of more than 1800 OCT images (including 308 images from stented segments) derived from 20 patients. Our major aim was to test our algorithms in images encountered in real-life conditions, with branches of different caliber, presence of blood, thrombus, stent struts, OCT wire artifacts and 
Table 4

Comparison of fully-automated and semi-automated vs. manual segmentation in stented segments $(\mathrm{n}=308$ images).

\begin{tabular}{|c|c|c|c|}
\hline & & $\begin{array}{l}\text { Fully-automated } \\
\text { vs. manually }\end{array}$ & $\begin{array}{l}\text { Semi-automated } \\
\text { vs. manually }\end{array}$ \\
\hline \multirow[t]{3}{*}{ Area $\left(\mathrm{mm}^{2}\right)$} & Bias & 0.2 & 0.1 \\
\hline & Upper limit & 1.5 & 1.2 \\
\hline & Lower limit & -1.1 & -1.0 \\
\hline \multirow{3}{*}{ Perimeter (mm) } & Bias & 0.8 & 0.5 \\
\hline & Upper limit & 3.0 & 1.9 \\
\hline & Lower limit & -1.4 & -0.8 \\
\hline \multirow{3}{*}{ Max radius (mm) } & Bias & 0.1 & 0.0 \\
\hline & Upper limit & 1.4 & 0.4 \\
\hline & Lower limit & -1.2 & -0.3 \\
\hline \multirow{3}{*}{ Min radius (mm) } & Bias & 0.0 & 0.0 \\
\hline & Upper limit & 0.3 & 0.2 \\
\hline & Lower limit & -0.3 & -0.2 \\
\hline \multirow[t]{3}{*}{ Max Diameter $(\mathrm{mm})$} & Bias & 0.1 & 0.0 \\
\hline & Upper limit & 0.9 & 0.6 \\
\hline & Lower limit & -0.7 & -0.5 \\
\hline \multirow[t]{3}{*}{ Min Diameter (mm) } & Bias & 0.0 & 0.0 \\
\hline & Upper limit & 0.3 & 0.3 \\
\hline & Lower limit & -0.2 & -0.2 \\
\hline \multirow[t]{3}{*}{ Centroid $\mathrm{x}(\mathrm{mm})$} & Bias & 0.9 & 0.1 \\
\hline & Upper limit & 0.4 & 0.3 \\
\hline & Lower limit & -0.2 & -0.1 \\
\hline \multirow[t]{3}{*}{ Centroid y (mm) } & Bias & 0.0 & 0.0 \\
\hline & Upper limit & 0.2 & 0.2 \\
\hline & Lower limit & -0.2 & -0.1 \\
\hline
\end{tabular}

heart motion artifacts. In a relatively small portion of images $(n=241$, $14 \%$ ) with the abovementioned artifacts we had to perform manual corrections of the automated segmentation without substantially compromising the analysis time. The study showed that our fullyautomated algorithm, as well as its semi-automated variation, performs very well enabling rapid and accurate segmentation of OCT images. Notably, our segmentation algorithm was effective and accurate even in stented areas.

\subsection{Performance of our algorithm in the context of the existing literature}

Limited research efforts have been focused on the development of an accurate and fast algorithm for automated segmentation of OCT images. A semi-automated method involving edge detection and smoothing was introduced showing satisfactory interobserver agreement [12]. A fully-automated OCT lumen contour detection method was also reported showing good quantitative results and satisfactory tracings in $97 \%$ of the cases [13]. Automated OCT segmentation techniques were also used to evaluate endothelialization and neointima formation following stent placement $[8,14,15]$. Those algorithms either employed edge detection with ad hoc thresholding or combined models with active or spline deformable intensity-based contours. However, it is still unclear how these methods can handle the bright concentric rings, which compromise the active contour model performance. More recently, two further automated OCT segmentation algorithms were developed and validated against manual segmentation in clinical OCT datasets, yielding satisfactory results $[16,17]$. On top of that, it was shown that the automated OCT segmentation is reproducible across different studies and shows good agreement with IVUS [18]. Similarly to the aforementioned studies our segmentation algorithm showed high accuracy in identifying the lumen-wall borders. Recently a novel OCT platform (ILUMIEN OPTIS PCI Optimization System, St. Jude Medical) was introduced capable of performing automated OCT segmentation. Direct comparison of our algorithm with this platform's algorithm was beyond the scope of the current study. In contrast to most of the previous studies, the validation dataset that was used in this study was quite extensive and representative of real world images. In addition, we utilized a global spectrum of validation metrics accounting for the vessel size (i.e. areas, radii and diameters) and shape (i.e. perimeter, centroid), thereby increasing the validity of our findings.

\subsection{Fully-automated vs. semi-automated segmentation of OCT images}

Developing a fully-automated image segmentation algorithm is challenging as medical images incorporate several motion- and image acquisition-related artifacts. In this study our fully-automated algorithm showed a good performance in $86 \%$ of images. In a subset of images (14\%) with stents or side branches the fully-automated algorithm did not perform optimally, resulting in a significantly increased relative error compared to manual segmentation (Fig. 6). To address the suboptimal segmentation of the fully-automated approach we applied manual modifications of the non-correctly segmented images (semi-automated approach). The agreement between that semi-automated segmentation and manual segmentation was comparable to the inter-observer agreement of the manual segmentation by two OCT experts, suggesting that the semiautomated algorithm may replace manual segmentation, restricting the human effort for inspection and limited manual adjustments. Of note, the analysis time with the semi-automated technique was not substantially prolonged compared to the fully-automated segmentation. Collectively, the semi-automated segmentation was a limited modification of the fully-automated approach which in real-life cannot be $100 \%$ accurate.

\subsection{Study limitations}

There are several limitations within the current study. First, we had to exclude 130 images from the initial dataset due to poor image quality mostly related to the presence of blood or clot. By consensus those images were non-interpretable and manual segmentation was not feasible (Supplemental Fig. 1). Second, small image artifacts, such as motioninduced artifacts, presence of small amount of blood or thrombus and side branches did not allow us to perform optimal fully-automated segmentation in 241 (14\%) images. In contrast to the 130 excluded poor quality images, manual segmentation was feasible in those 241 images with artifacts. Other studies also showed that $3-15 \%$ of the automatically segmented images require manual adjustment $[13,18]$. This set of manually corrected images could set the basis for further algorithm improvement. Third, we did not segment the entire OCT pullback from each patient but we selected one every two or three images per patient. Automated segmentation of OCT images will be incorporated in a future complete tool performing OCT-based 3D reconstruction of the coronary arteries. In this tool periodic selection of OCT images is anticipated to account for electrocardiographic gating.

\subsection{Clinical perspectives and future work}

Our algorithm could facilitate OCT segmentation for clinical and research purposes. In the clinical arena, there is an increasing demand for longitudinal clinical studies to assess the natural history of atherosclerosis, as well as the impact of therapeutic interventions $[1,3,19]$. OCT imaging can play a key role in such studies highlighting the need for developing automated segmentation algorithms that can rapidly and effectively perform lumen morphometric analysis. Furthermore, OCT has been pivotal in assessing stent strut apposition and endothelialization $[5,7,8]$. Automated segmentation algorithms like the one we present in the current study are anticipated to facilitate quantitative analysis in longitudinal studies investigating stent restenosis and thrombosis.

Studies have underscored the role of endothelial shear stress in coronary artery atherosclerosis. Endothelial shear stress calculation in 
A

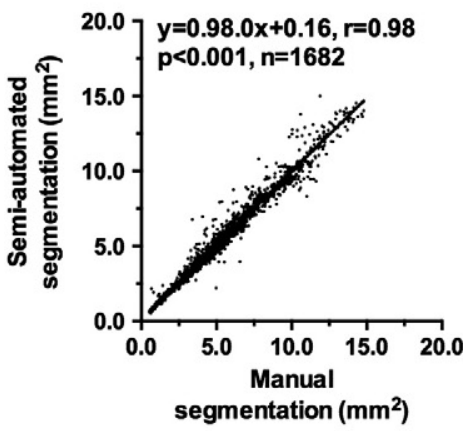

Max radius

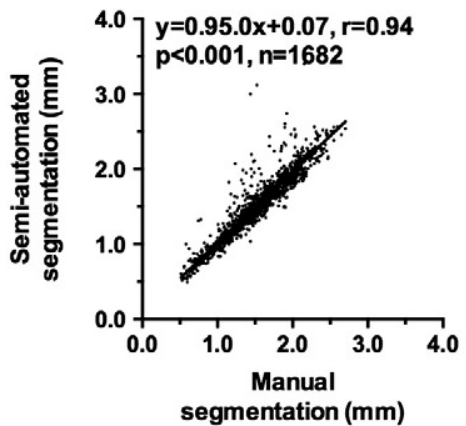

Max diameter

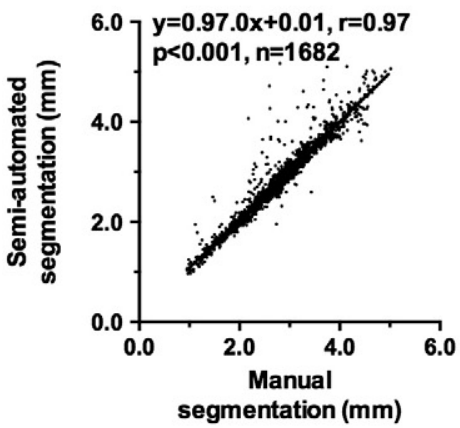

Centroid $\mathbf{x}$

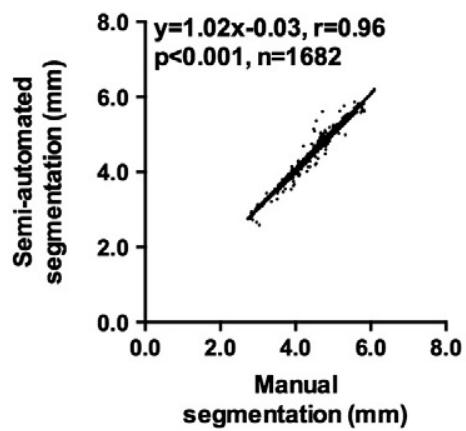

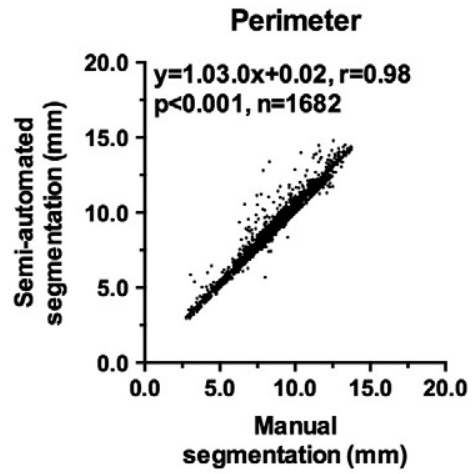

Min radius

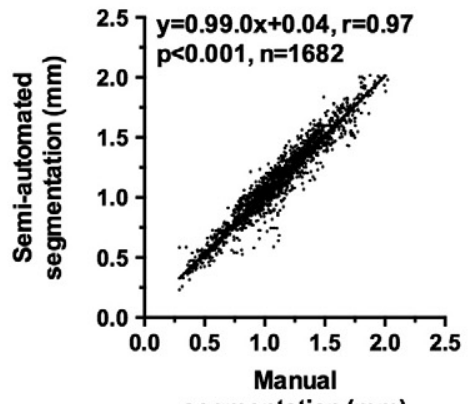

segmentation $(\mathbf{m m})$

\section{Min diameter}

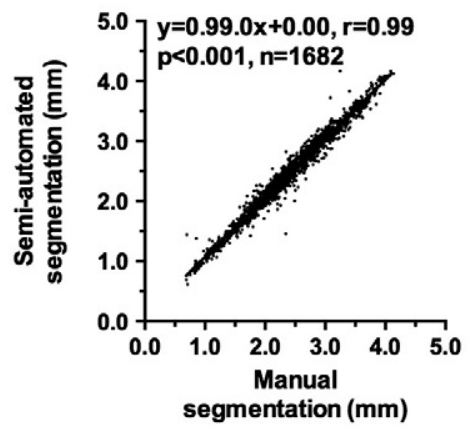

Centroid y

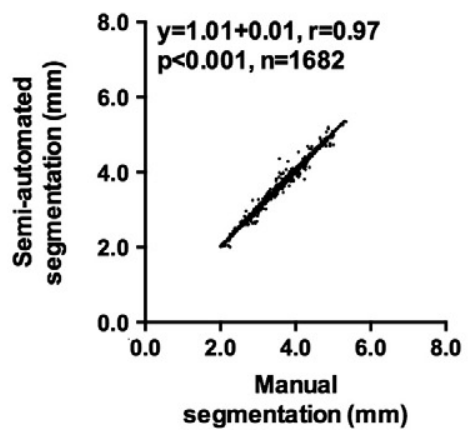

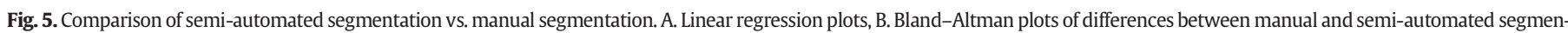

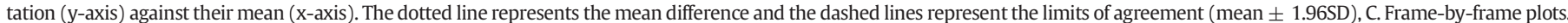

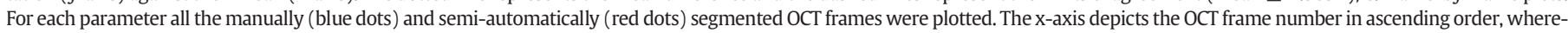
as the $\mathrm{y}$-axis shows the corresponding variable. Note that the OCT images in $\mathrm{x}$-axis are distributed independently of patient, type of artery and location.

man can be achieved through computational fluid dynamics of 3D reconstructed coronary arteries [20-22]. To date, 3D reconstruction was based in IVUS and coronary CT angiography [23]. OCT could be also utilized in the 3D reconstruction algorithms enabling simultaneous assessment of functional (i.e. endothelial shear stress) and pathobiologic (i.e. inflammation, lipid pool) plaque features [9] [24,25]. The proposed 


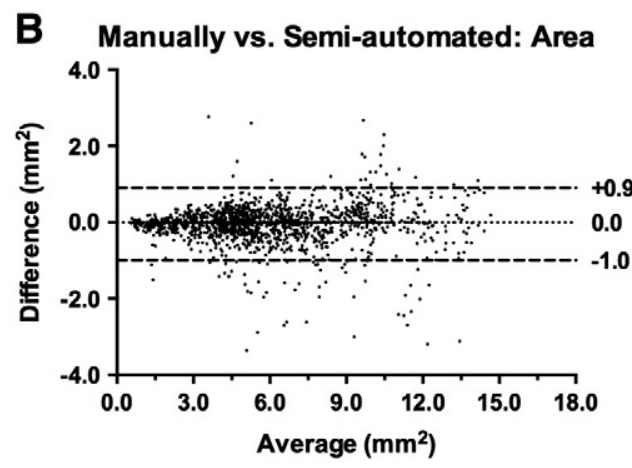

Manually vs. Semi-automated: Max radius

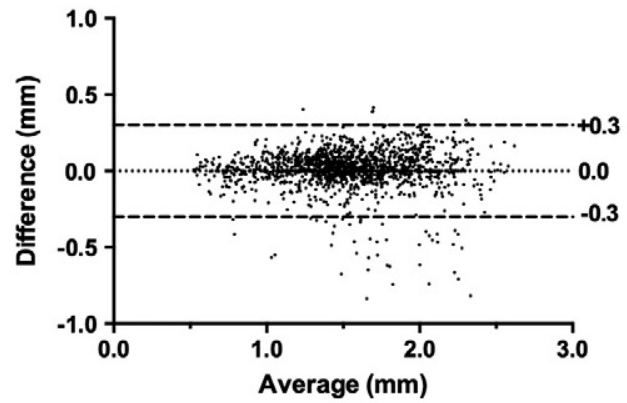

Manually vs. Semi-automated: Max diameter

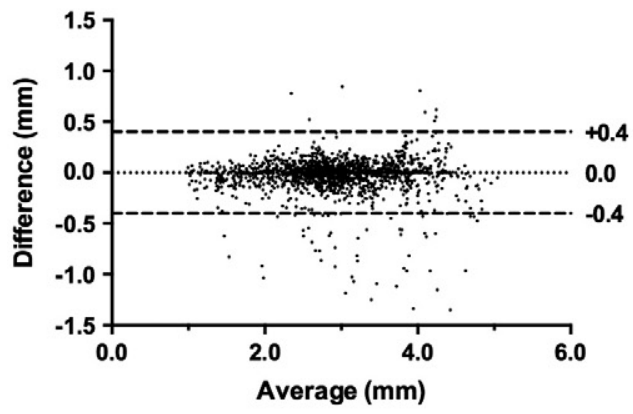

Manually vs. Semi-automated: Centroid $\mathbf{x}$

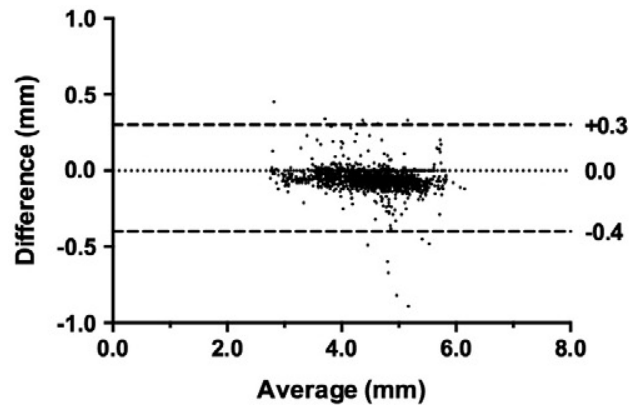

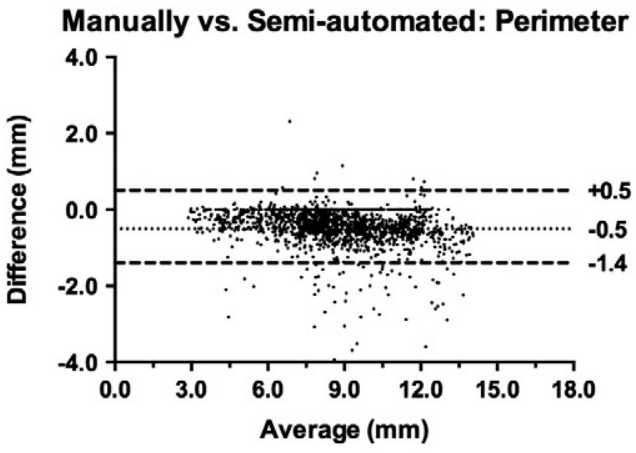

Manually vs. Semi-automated: Min radius

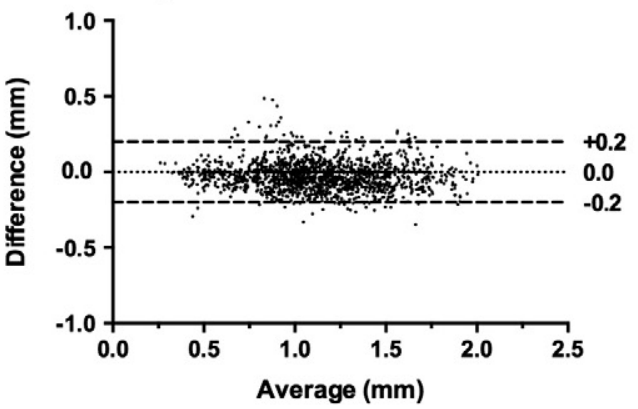

Manually vs. Semi-automated: Min diameter

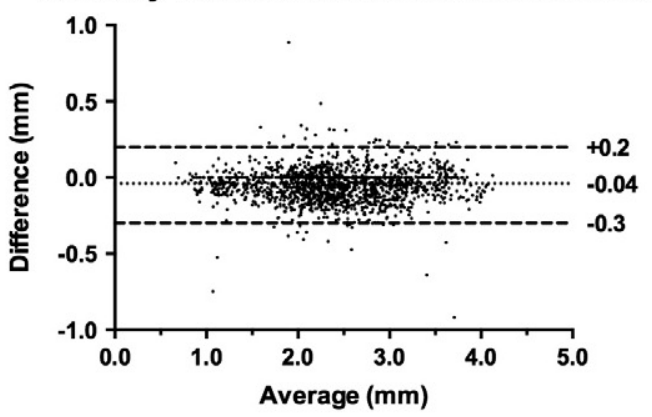

Manually vs. Semi-automated: Centroid y

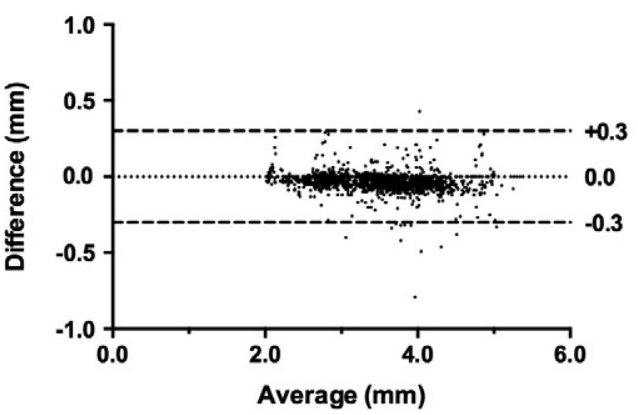

Fig. 5 (continued).

segmentation algorithm represents a key step towards fast and robust OCT-based geometrically-correct 3D reconstruction of coronary arteries.

Our future work involves the: (i) refinement of our algorithm to increase its robustness in regions with image artifacts, (ii) development of a user-friendly graphical user interface that will allow routine OCT analyses with the option of manual correction as needed and export of the quantitative results, and (iii) incorporation of the automated segmentation algorithm into a complete 3D coronary artery reconstruction suite which will allow computational fluid dynamic studies [21].

\section{Conclusions}

In the current work we presented a fully-automated algorithm, as well as a semi-automated variation of it, that enabled rapid and reliable segmentation of intracoronary OCT images. Our algorithm was extensively validated in a "real-life" dataset of OCT images 


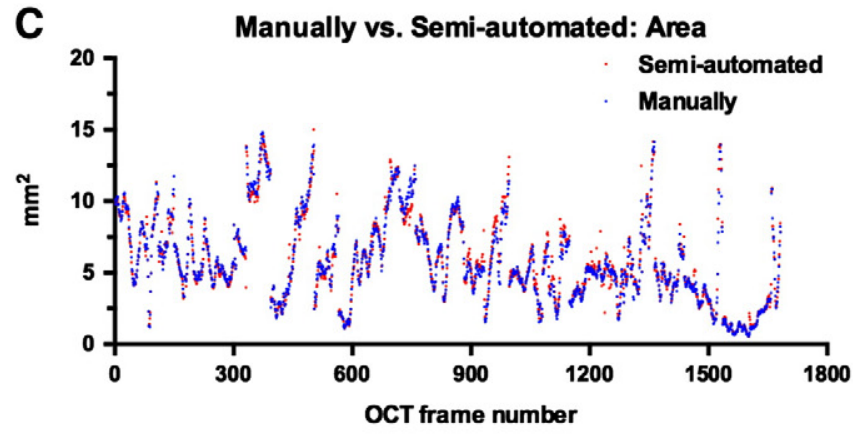

Manually vs. Semi-automated: Max radius

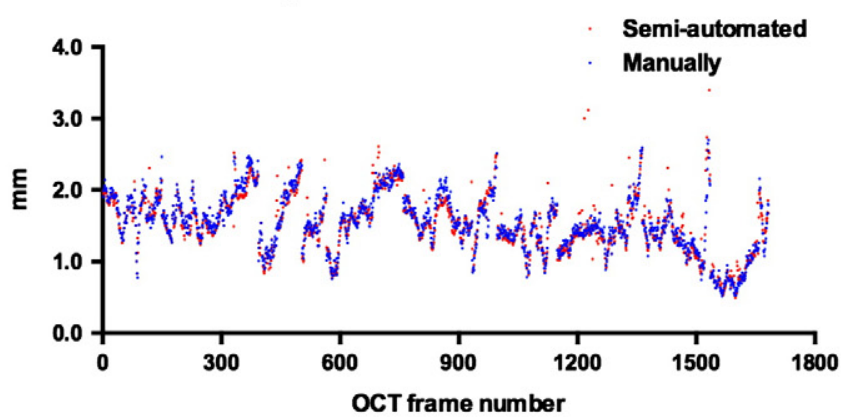

Manually vs.Semi-automated: Max diameter

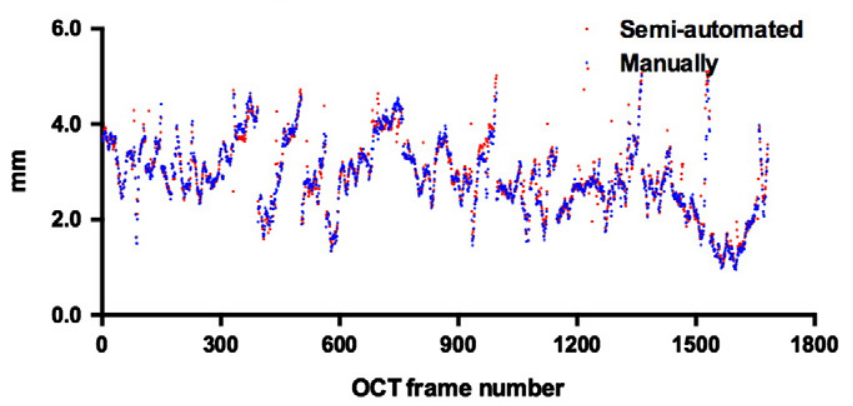

Manually vs. Semi-automated: Centroid $\mathbf{x}$

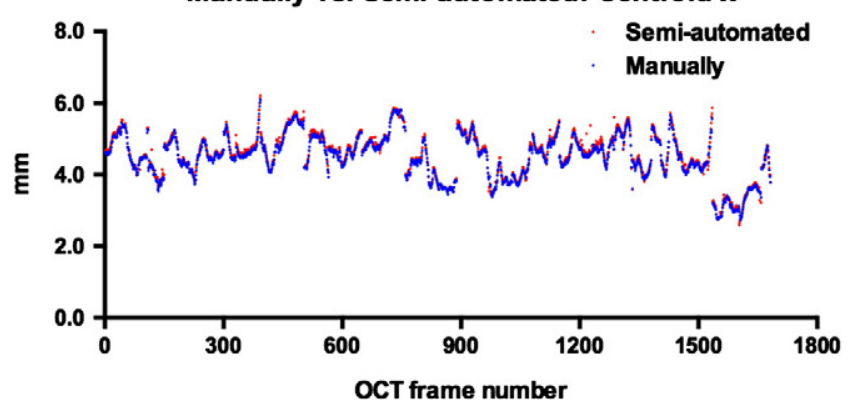

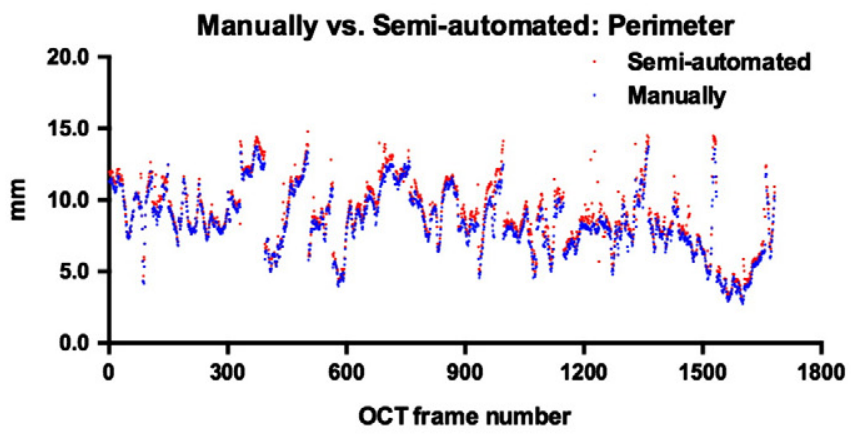

Manually vs.Semi-automated: Min radius

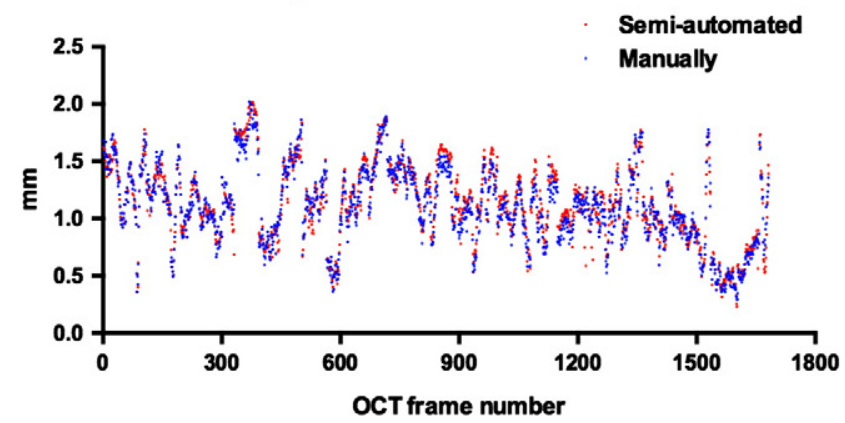

Manually vs.Semi-automated: Min diameter

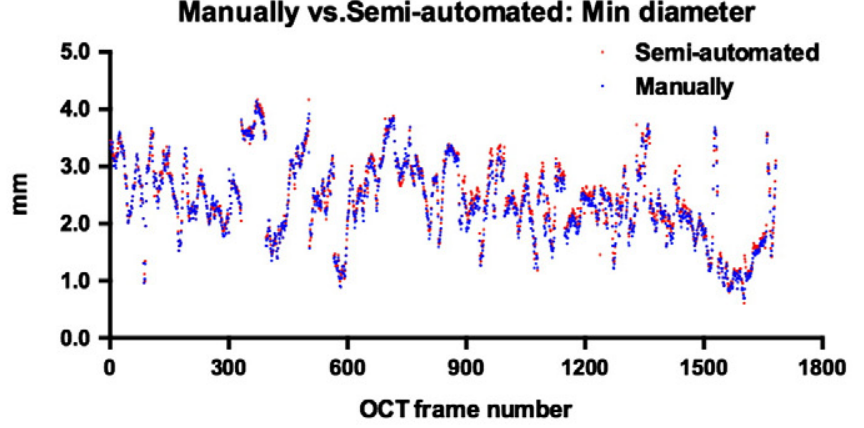

Manually vs. Semi-automated: Centroid y

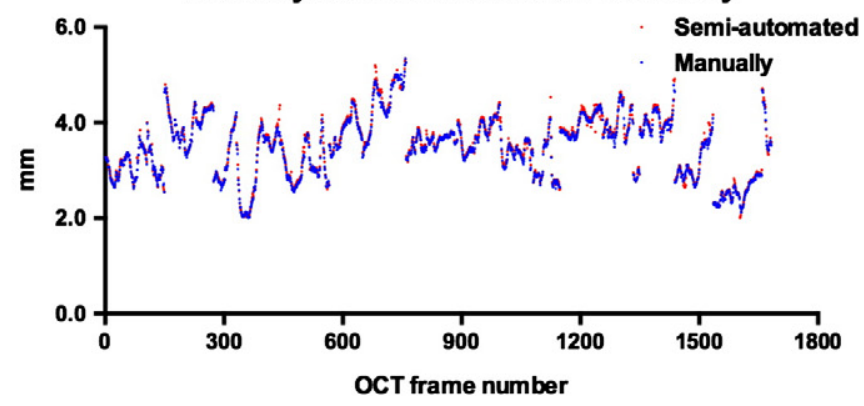

Fig. 5 (continued).

demonstrating that it is feasible and accurate requiring limited manual interventions. This algorithm has the potential to evolve to a useful clinical and research tool facilitating plaque quantitative and local hemodynamic analyses in OCT studies.
Funding sources

European Commission, Marie Curie International Reintegration Grant, Project: SMILE (number: 249303); General Secretariat of Research and

Table 5

Time gain of fully-automated and semi-automated segmentation vs. manual.

\begin{tabular}{|c|c|c|c|c|c|c|c|}
\hline & Manually & Fully-automated segmentation & Time gain vs. manually & $p$ & Semi-automated segmentation & Time gain vs. manually & $p$ \\
\hline Time (s/image) & 30 & $<1$ & $>97 \%$ & $<0.001$ & $<5.1$ & $>83 \%$ & $<0.001$ \\
\hline
\end{tabular}




\section{Correct fully-automated segmentation}
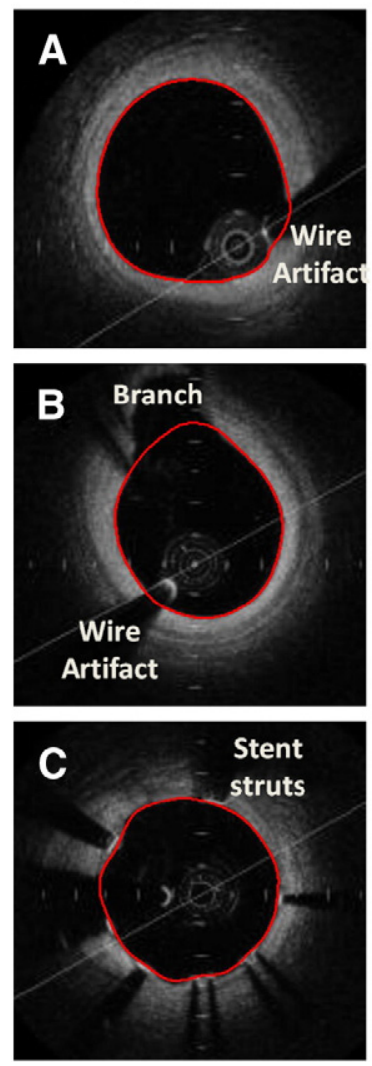

Manual segmentation
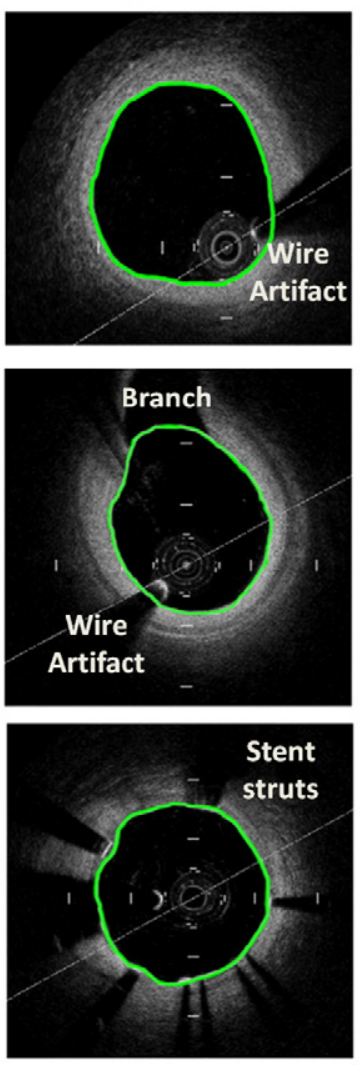

Suboptimal fullyautomated segmentation
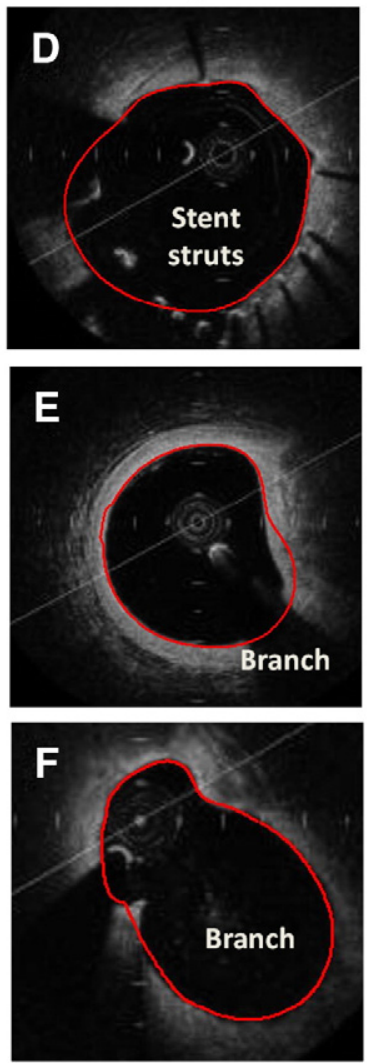

Manual segmentation
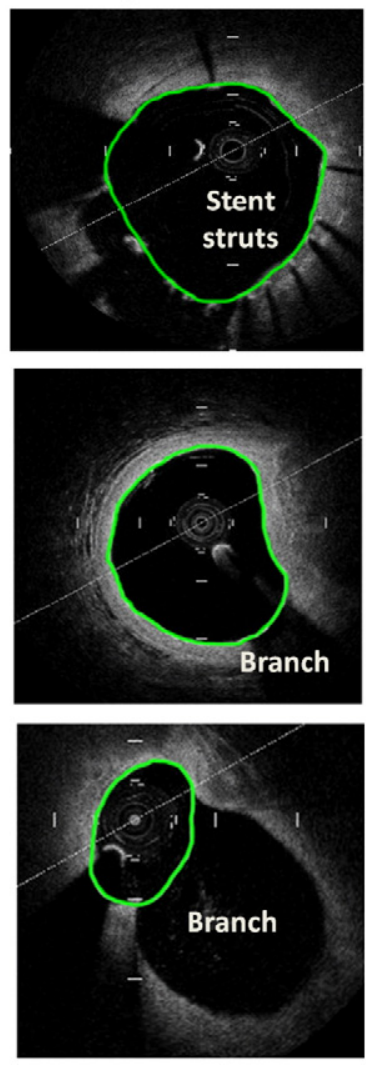

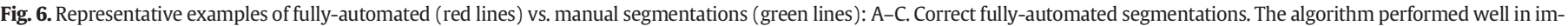

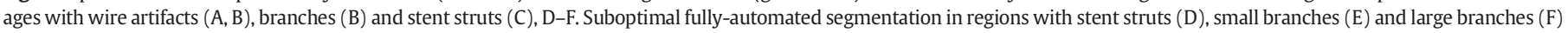
requiring manual corrections.

Technology, Program: Heracleitus II, Athens, Greece; Behrakis Foundation, Boston, USA.

\section{Appendix A. Supplementary data}

Supplementary data to this article can be found online at http://dx. doi.org/10.1016/j.ijcard.2014.01.071.

\section{References}

[1] Bezerra HG, Costa MA, Guagliumi G, Rollins AM, Simon DI. Intracoronary optical coherence tomography: a comprehensive review clinical and research applications. JACC Cardiovasc Imaging 2009;2:1035-46.

[2] Prati F, Regar E, Mintz GS, et al. Expert review document on methodology, terminol ogy, and clinical applications of optical coherence tomography: physical principles, methodology of image acquisition, and clinical application for assessment of coronary arteries and atherosclerosis. Eur Heart J 2010;31:401-15.

[3] Brezinski ME. Current capabilities and challenges for optical coherence tomography as a high-impact cardiovascular imaging modality. Circulation 2011;123:2913-5.

[4] Tearney GJ, Yabushita H, Houser SL, et al. Quantification of macrophage content in atherosclerotic plaques by optical coherence tomography. Circulation 2003;107:113-9.

[5] Barlis P, Regar E, Serruys PW, et al. An optical coherence tomography study of a biodegradable vs. durable polymer-coated limus-eluting stent: a LEADERS trial substudy. Eur Heart J 2010;31:165-76.

[6] Gonzalo N, Garcia-Garcia HM, Regar E, et al. In vivo assessment of high-risk coronary plaques at bifurcations with combined intravascular ultrasound and optical coherence tomography. JACC Cardiovasc Imaging 2009;2:473-82.

[7] Gonzalo N, Serruys PW, Okamura T, et al. Optical coherence tomography patterns of stent restenosis. Am Heart J 2009;158:284-93.

[8] Unal G, Gurmeric S, Carlier SG. Stent implant follow-up in intravascular optical coherence tomography images. Int J Cardiovasc Imaging 2010;26:809-16.

[9] Giannopoulos A, Chatzizisis YS, Giannoglou GD. Optical coherence tomography: an arrow in our quiver. Expert Rev Cardiovasc Ther 2012;10:539-41.
[10] Papadogiorgaki M, Mezaris V, Chatzizisis YS, Giannoglou GD, Kompatsiaris I. Image analysis techniques for automated IVUS contour detection. Ultrasound Med Biol 2008;34:1482-98.

[11] Bland JM, Altman DG. Statistical methods for assessing agreement between two methods of clinical measurement. Lancet 1986;1:307-10

[12] Tanimoto S, Rodriguez-Granillo G, Barlis P, et al. A novel approach for quantitative analysis of intracoronary optical coherence tomography: high inter-observer agreement with computer-assisted contour detection. Catheter Cardiovasc Interv 2008;72:228-35

[13] Sihan K, Botha C, Post F, et al. Fully automatic three-dimensional quantitative analysis of intracoronary optical coherence tomography: method and validation. Catheter Cardiovasc Interv 2009;74:1058-65.

[14] Bonnema GT, Cardinal KO, Williams SK, Barton JK. An automatic algorithm for detecting stent endothelialization from volumetric optical coherence tomography datasets. Phys Med Biol 2008:53:3083-98.

[15] Kauffmann C, Motreff P, Sarry L. In vivo supervised analysis of stent reendothelialization from optical coherence tomography. IEEE Trans Med Imaging 2010;29:807-18

[16] Tsantis S, Kagadis GC, Katsanos K, Karnabatidis D, Bourantas G, Nikiforidis GC. Automatic vessel lumen segmentation and stent strut detection in intravascular optical coherence tomography. Med Phys 2012;39:503-13.

[17] Ughi GJ, Adriaenssens T, Onsea K, et al. Automatic segmentation of in-vivo intracoronary optical coherence tomography images to assess stent strut apposition and coverage. Int J Cardiovasc Imaging 2012;28:229-41.

[18] Jamil Z, Tearney G, Bruining N, et al. Interstudy reproducibility of the second generation, fourier domain optical coherence tomography in patients with coronary artery disease and comparison with intravascular ultrasound: a study applying automated contour detection. Int J Cardiovasc Imaging 2013;29:39-51.

[19] Jang IK, Bouma BE, Kang DH, et al. Visualization of coronary atherosclerotic plaques in patients using optical coherence tomography: comparison with intravascular ultrasound. J Am Coll Cardiol 2002;39:604-9.

[20] Chatzizisis YS, Coskun AU, Jonas M, Edelman ER, Feldman CL, Stone PH. Role of endothelial shear stress in the natural history of coronary atherosclerosis and vascular remodeling: molecular, cellular, and vascular behavior. J Am Coll Cardiol 2007;49:2379-93. 
[21] Stone PH, Saito S, Takahashi S, et al. Prediction of progression of coronary artery disease and clinical outcomes using vascular profiling of endothelial shear stress and arterial plaque characteristics: the PREDICTION study. Circulation 2012;126:172-81.

[22] Wentzel JJ, Chatzizisis YS, Gijsen FJ, Giannoglou GD, Feldman CL, Stone PH. Endothelial shear stress in the evolution of coronary atherosclerotic plaque and vascular remodelling: current understanding and remaining questions. Cardiovasc Res 2012;96:234-43.

[23] Doulaverakis C, Tsampoulatidis I, Antoniadis AP, Chatzizisis YS, Giannopoulos A, Kompatsiaris I, Giannoglou GD. IVUSAngio tool: a publicly available software for fast and accurate 3D reconstruction of coronary arteries. Comput Biol Med 2012;43(11):1793-803.

[24] Toutouzas K, Riga M, Giannopoulos A, et al. Geometrically-correct 3D OCT: proof of concept, methodology and first-in-man validation study. Eur Heart J (Abstract supplement) 2012;33:1.

[25] Giannopoulos A, Toutouzas K, Riga M, et al. Plaques with increased lipid core and thin fibrous cap occur in coronary artery regions with low endothelial shear stress: a 3D optical coherence tomography pilot study in human. Eur Heart J (Abstract supplement) 2012;33:2. 